\title{
Surgical Management of Closed Head Injury
}

Ahmed Swailam Mohamed*, Mahmoud Mahmoud El Hendawy, Shymaa Adel Ismail

Department of Neurosurgery, Faculty of Medicine for girls Al-Azhar University

*Corresponding author: Ahmed Swailam Mohamed, Mobile: (+20)01064174242, E-Mail: ahmed.swailam.f.n.s.@gmail.com

\begin{abstract}
Background: Closed-head injury is a type of traumatic brain injury in which the skull and dura mater remain intact. Closed-head injuries are the leading cause of death in children under 4 years old and the most common cause of physical disability and cognitive impairment in young people.

Objective: The study aimed to evaluate cases of closed head injury as regard clinical picture, investigations, surgical management, outcome, and prognosis.

Patient and methods: A prospective study was conducted on Sixty Two patients, twenty two of them were subjected to surgery. Many preoperative factors were studied to evaluate the prognosis of such cases.

Results: Among 62 cases, 22 cases were submitted to surgery 17 extradural hematomas (EDH) were submitted to surgery, some associated with sub arachnoid hemorrhage (SAH) and acute subdural hematomas (SDH).

Conclusion: Early diagnosis and management of primary lesion of closed head injury is very important to prevent secondary brain injury.
\end{abstract}

Keywords: Glasgow coma scale, post-concussion syndrome, Computerized tomography, Traumatic brain injury.

\section{INTRODUCTION}

Closed head injuries are classified as either primary or secondary. A primary injury results from the initial anatomical and physiological insult. A secondary injury results from hypotension, hypoxia, acidosis, edema, or other subsequent factors that can secondarily damage brain tissue. The major focus in the management of acute closed head injury is the prevention of secondary injuries and the preservation of neurological functions that are not damaged by the primary injury ${ }^{(\mathbf{1})}$.

The goal of imaging in the management of head trauma is to identify treatable injuries to prevent secondary damage. CT continues to be the modality of choice in the evaluation of acute head injury. CT is preferred in the acute setting because it is fast, is widely available and can easily accommodate life-support and monitoring equipment. CT can accurately identify space-occupying lesions, acute hemorrhages, mass effect, midline shift, hydrocephalus, ischemia, and herniation. MRI is indicated for patients with acute traumatic brain injury (TBI) when the neurologic findings are unexplained by CT. MRI is also the modality of choice for subacute or chronic injury. Advances in MR methods, such as diffusion-weighted imaging further improve the neuroradiological evaluation of traumatic brain injury and enhance our understanding of the pathophysiological manifestations of head trauma ${ }^{(2)}$.

\section{PATIENTS AND METHODS}

A prospective study was conducted on Sixty Two patients who came to Luxor International Hospital and Al-Zahraa University Hospital from November 2018 to November 2019. "Only 22 cases i.e. $35 \%$ of the patients had surgery while the rest $65 \%$ had conservative management". (NB: written consents were taken from all patients).

The patients included in the study: All patients with closed head injury who were submitted to surgical interference including patients with extradural hematomas, patients with subdural hematomas, patients with cerebral contusion, patients with cerebral hemorrhage, patients with tnterventricular hemorrhage and patients with sub arachnoid hemorrhage.

Patients with open head injury and closed head injury that were treated conservatively were excluded from the study.

All patients were subjected to first aid measures, full general and neurological examination, primary survey (abdominal u/s, pelvis x-ray, chest x-ray, and CT brain), carful follow up of the patient and his neurological status as well as scheduled CT brain for the patients for follow up according to their neurological condition. Finally, surgical intervention for the indicated cases which was by one of two procedures; craniotomy mainly for $\mathrm{EDH}$ and acute SDH and bur hole for subacute SDH. Patients are asked to come back to hospital if they experience any of symptoms like headache, vomiting, manifestation of increased intracranial tension, newly developed weakness and convulsion, otherwise scheduled follow up of the pt. in outpatient clinic (OPC) at 1 week after discharge, then follow up radiologically and clinically.

\section{Ethical approval}

An approval of the study was obtained from AlAzhar University academic and ethical committee. Every patient signed an informed written consent for acceptance of the operation. 


\section{Statistical analysis}

Recorded data were analyzed using the statistical package for social sciences, version 20.0 (SPSS Inc., Chicago, Illinois, USA). Quantitative data were expressed as mean \pm standard deviation (SD). Qualitative data were expressed as frequency and percentage.

\section{The following tests were done:}

- Independent-samples t-test of significance was used when comparing between two means.

- Chi-square $\left(\mathrm{x}^{2}\right)$ test of significance was used in order to compare proportions between two qualitative parameters.

- The confidence interval was set to $95 \%$ and the margin of error accepted was set to $5 \%$. The p-value was considered significant as the following:

- Probability (P-value)

- P-value <0.05 was considered significant. - P-value $<0.001$ was considered as highly significant. - P-value $>0.05$ was considered insignificant.

\section{RESULTS}

A total of 62 cases, 22 surgical cases of closed head injury that were admitted during the period of November 2018 - November 2019 were included in this study. Of the 22 Cases included in the study, the majority were in the age group of (0-20) and (21-40) years, i.e. $50 \%$ for the first age group $(n=11)$ and $32 \%$ for the second group $(\mathrm{n}=7)$. Among all the patients $73 \%(\mathrm{n}=16)$ were males and $27 \%(n=6)$ were females shown in (figures $1 \& 2$ ).

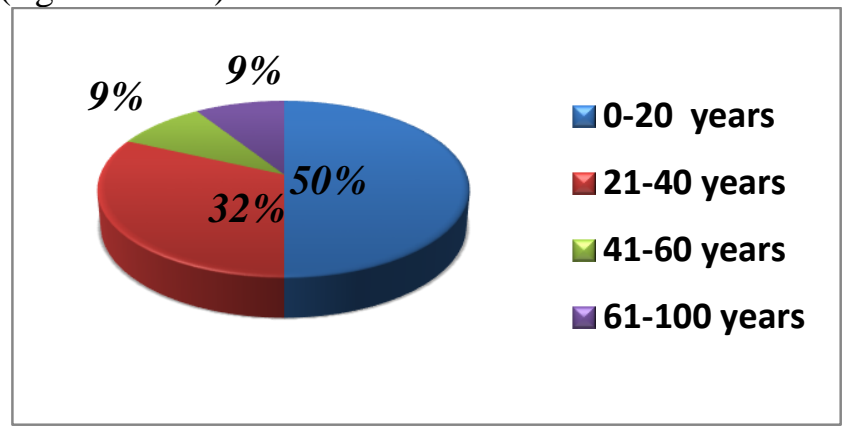

Figure (1): Age distribution of the studied cases.

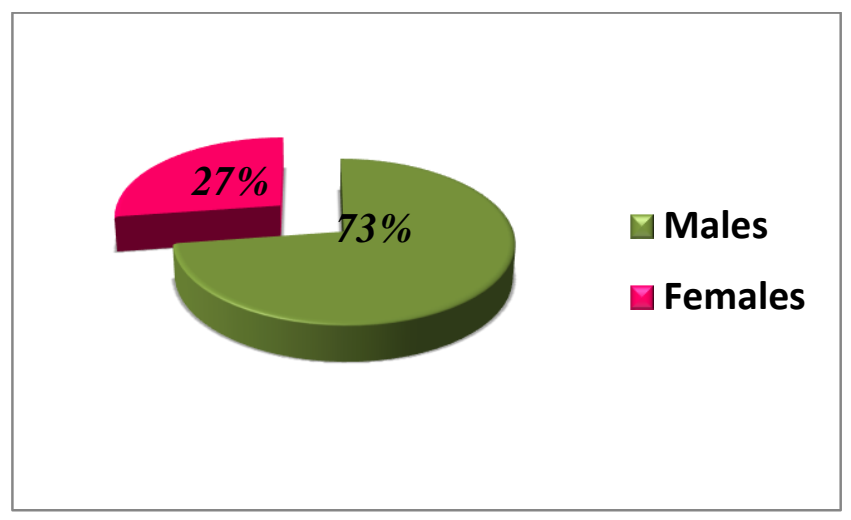

Figure (2): Gender distribution of the studied cases.
Pre and post-Operative Glasgow Coma Scale (GCS) and Pupil Reaction distribution of the studied cases:

In this part of the study, the patients were divided into two main groups; pre- and post-operative groups. Each group was divided into six sub groups as shown in table (1). The post-operative group was classified only into two sub groups.

On the other hand according to pupil reaction, pts. were divided into two groups pre- and postoperative groups and each group was divided into two sub groups; reactive pupil and dilated fixed pupil as shown in table (2). The post-operative groups were as follows: 21 pts. (95.5\%) with reactive pupil and only one pt. (4.5\%) with dilated fixed pupil (NB: this case was represented by massive EDH and brain edema that led to brain herniation and death).

Table 1: Pre and post-operative GCS outcome

\begin{tabular}{|l|l|l|}
\hline & $\begin{array}{c}\text { Pre-operative } \\
\text { cases No. and } \\
\%\end{array}$ & $\begin{array}{c}\text { Post-operative } \\
\text { cases No. and } \\
\%\end{array}$ \\
\hline GCS 15 & 5 pt. $=23 \%$ & 21 pt. $=95.5 \%$ \\
\hline GCS 14 & 6 pt. $=27 \%$ & 0 pt. $=0 \%$ \\
\hline GCS 13 & 4 pt. $=18 \%$ & 0 pt. $=0 \%$ \\
\hline GCS 12 & $5 \mathrm{pt} .=23 \%$ & 0 pt. $=0 \%$ \\
\hline GCS 11 & $1 \mathrm{pt} .=4.5 \%$ & $0 \mathrm{pt} .=0 \%$ \\
\hline GCS $<8$ & $1 \mathrm{pt} .=4.5 \%$ & $1 \mathrm{pt} .=4.5 \%$ \\
\hline Total & $22 \mathrm{pt} .=100 \%$ & $22 \mathrm{pt} .=100 \%$ \\
\hline
\end{tabular}

Table 2: Pre and post-operative pupil reaction outcome

\begin{tabular}{|l|l|l|}
\hline & $\begin{array}{c}\text { Pre-operative } \\
\text { cases No. and } \\
\%\end{array}$ & $\begin{array}{c}\text { Post-operative } \\
\text { cases No. and } \\
\%\end{array}$ \\
\hline Reactive pupil & $22 \mathrm{pt} .=100 \%$ & $21 \mathrm{pt} .=95.5 \%$ \\
\hline $\begin{array}{l}\text { Dilated fixed } \\
\text { pupil }\end{array}$ & $0 \mathrm{pt} .=0 \%$ & $1 \mathrm{pt} .=4.5 \%$ \\
\hline Total & $22 \mathrm{pt} .=100 \%$ & $22 \mathrm{pt} .=100 \%$ \\
\hline
\end{tabular}

\section{Post-concussion syndrome:}

Post-concussion syndrome was positive in 19 cases $(86.36 \%)$ of the studied 22 cases in the form of headache (18 cases i.e. $81.81 \%$ ), vertigo (15 cases i.e. $68 \%$ ) and loss of consciousness (LOC) at time of trauma (19 cases i.e. $86.36 \%)$ that were divided as follows: 15 out of 19 pts. $(79 \%)$ were $\leq 10$ minutes, 4 out of the 19 studied cases $(21 \%)$ were $\geq 10$ minutes shown in table (3). In addition to dizziness (20 cases i.e. $90.90 \%$ ). 
Table 3: Period of LOC at time of trauma for cases of PCS

\begin{tabular}{|c|c|c|c|c|}
\hline $\begin{array}{c}\leq \mathbf{1 0} \\
\text { mins }\end{array}$ & $\boldsymbol{\%}$ & $\geq \mathbf{1 0}$ mins & $\boldsymbol{\%}$ & Total \\
\hline 17 & 77.27 & 5 & 22.72 & 22 \\
\hline
\end{tabular}

FU of post-concussion syndrome for each item of the previous groups was as follows:

The Headache group 12 pts. (66.66\%) of them lasted for two months then improved and 6 pts. (33.33 $\%)$ lasted for six months then improved. The vertigo group 14 pts. (93.33\%) improved after three months while only one pt. (6.6\%) took more than six months to improve. Dizziness group, 15 pts. $(75 \%)$ improved after about three months while 5 pts. $(25 \%)$ improved after six months.

Of the previous results we found that there were some relations among the non-improved pts. as follows:

All pts. with headache, dizziness and vertigo suffered long period of LOC at time of trauma $\geq 10$ minutes and lasted longer period of time for recovery than those of the same groups but with shorter time of LOC.

\section{Neurological deficit:}

Neurological deficit were 4 cases (18.18\%) one of them with grade four Rt. side weakness and the rest varied between grade two to three Lt. side weakness. Seizures were 8 cases $(36.3 \%)$. The Seizures group, 7 pts. $(87.5 \%)$ improved in the form of $\downarrow$ antiepileptic drugs doses after about six month while one pt. (12.5\%) didn't improve as the case still on antiepileptic drugs. The pt. with non-improved weakness presented with huge EDH compressing the matter area and came late to our hospital $6 \mathrm{hrs}$ after trauma (delayed surgical manner).

\section{Radiological finding:}

The majority of lesions were EDH (17 pts. i.e. $77.27 \%$ ), then SDH (5 cases i.e. $22.72 \%$ ) of the studied 22 cases and they were divided as follows: 3 pts $(60 \%)$ were acute SDH and 2 pts $(40 \%)$ were subacute SDH. EDH were associated with SAH in 4 pts. (23.5\%). EDH were also associated with brain edema in 10 pts. $(58.8 \%)$ and midline shift in 6 pt. (35.3\%) of the studied 17 cases.

SDH were associated with SAH in 2 pts. (40\%). Brain edema in 1 pt. (20\%) and midline shift in 1 pt. (20 $\%)$ of the studied 5 cases.

SAH presented in suprasellar cistern (4 pt. i.e. $18.18 \%$ ) all with EDH cases and in the sylvian fissure in 3 pts. (13.63\%) 2 of them with SDH and only 1 with EDH.

Midline shift was founded in 7 pts. (31.81\%) of the studied 22 cases. They were distributed as follows according to their amount: $3-4 \mathrm{~mm}$ in 3 pts. $(42.85 \%)$, $6-8 \mathrm{~mm}$ in 3 pts. $(42.85 \%)$ and $8-11 \mathrm{~mm}$ only in one pt. (14.28) of the studied 7 cases.

From the previous data we found that the GCS of cases associated with SAH midline shift and brain edema, ranged between 13 and 8 .

Post-operative follow up (FU) CT findings were as follows: all SDH CTs were free, while all FU CTs of $\mathrm{EDH}$ cases were also free except for one case, which died three days after operation, SAH FU CTs took about three months to improve except for 3 pts. with midline shift more than $6 \mathrm{~mm}$ where they took more than six months to improve.

\section{Surgical Procedures:}

Of the 22 studied cases, the majority of them submitted to craniotomy (20 pts. i.e. $90.90 \%)$ and 17 pts. $(85 \%)$ were $\mathrm{EDH}$, while 3 pt. $(15 \%)$ were acute $\mathrm{SDH}$. On the other hand, 2 pts. $(9.09 \%)$ were subacute SDH operated by burr hole technique (1 pt. done by single burr hole technique and 1 case done by double burr hole technique) as shown in table (4).

Table 4: Craniotomy and Burr hole techniques distribution.

\begin{tabular}{|l|l|l|l|}
\hline Craniotomy & $\%$ & Burr hole & \% \\
& & & \\
\hline 20 & 90.90 & & 9.09 \\
\hline
\end{tabular}

\section{Survival and mortality rates outcome:}

Of our studied cases the survived cases were 21 $(95.5 \%)$ cases while one case died $(4.5 \%)$ (Figure 3 ). From the previous data one of the causes of death was sever midline shift, which was more than $8.5 \mathrm{~mm}$ in this case as well as massive brain edema.

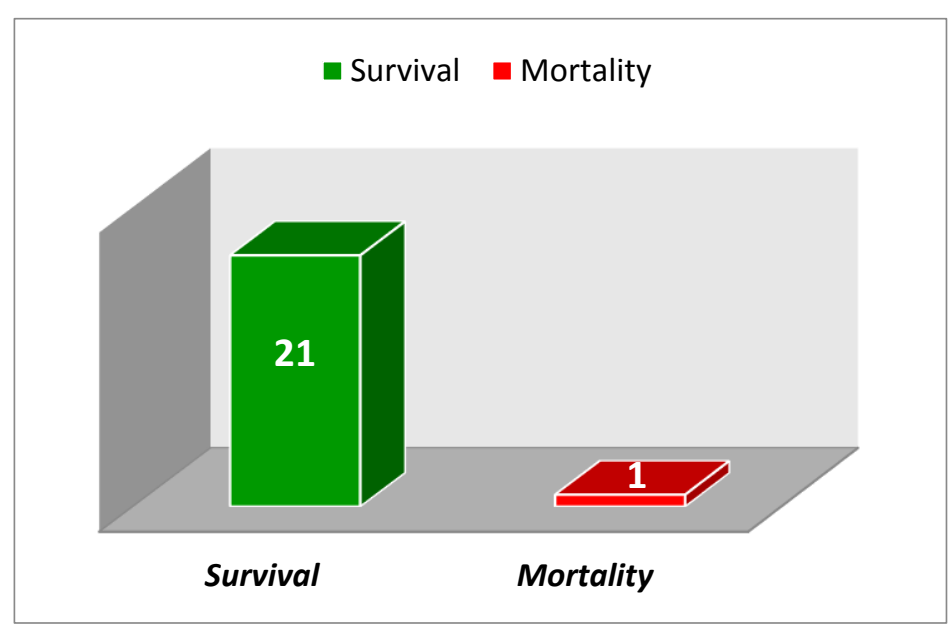

Figure (3): Mortality and Survival cases distribution. 


\section{Illustrative cases}

CASE 1

A 32 years old $\delta$ patient with history of head trauma as patient was kicked by animal (Buffalo). He suffered from loss of consciousness for about five minutes. He came to Emergency Room (ER) with GCS 14/15, repeated vomiting and both pupils were regular rounded and reactive. First aids were done to him, full labs $\mathrm{ABG}$ revealed abnormalities $\left(\uparrow \mathrm{PCO}_{2}\right.$ and $\left.\downarrow \mathrm{PH}\right)$. CT brain was done which revealed a massive right frontal EDH (Fig. 4) with $\geq 5 \mathrm{~mm}$ midline shift then patients were prepared for surgery. Evacuation of hematoma was done by craniotomy procedure.

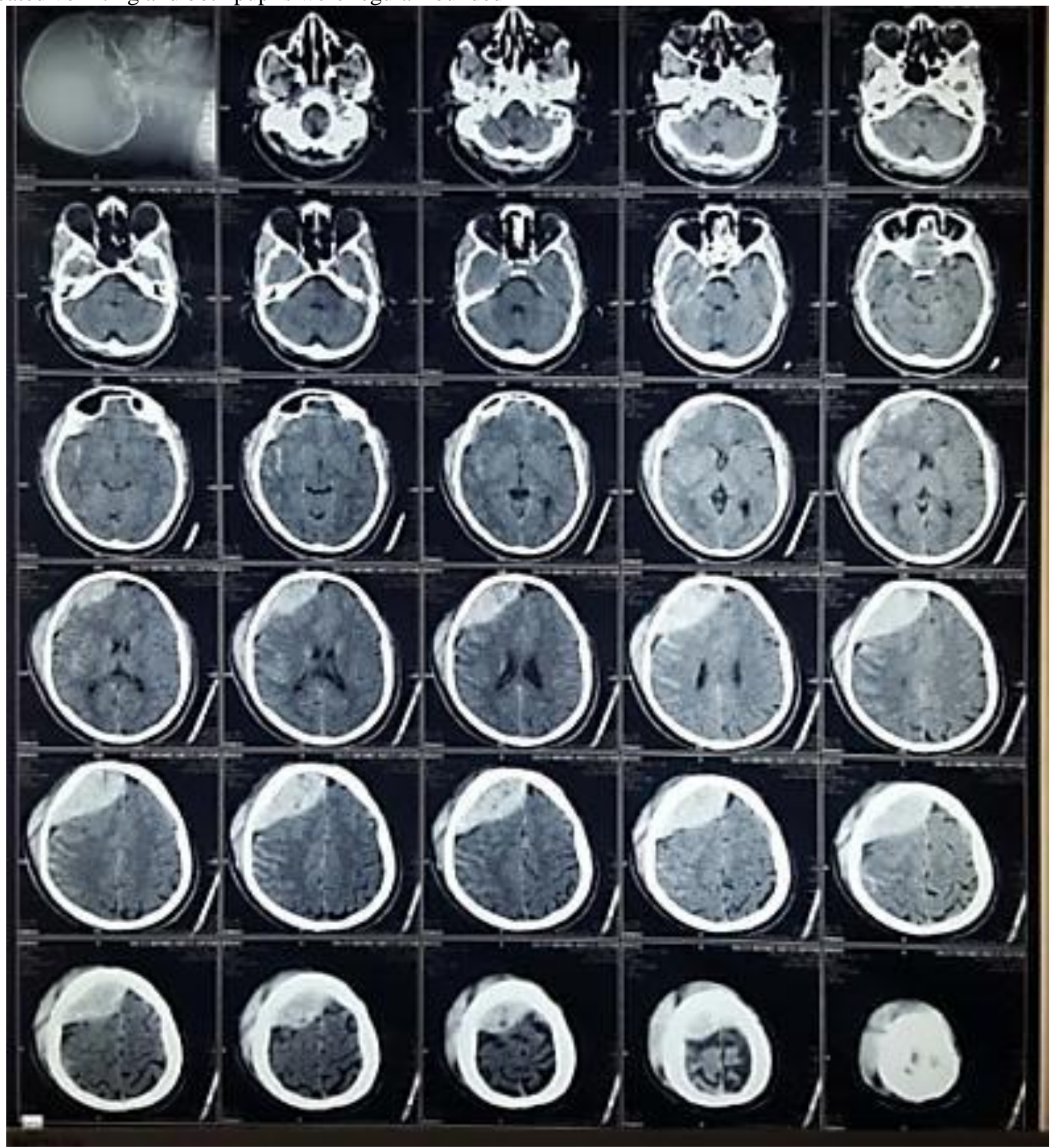

Figure (4): Pre-operative CT scan Rt. Frontal massive EDH.

After 24 hours follow up CT showed complete evacuation and no recollection. 2 days later patient was discharged fully conscious. One month later the patient visited our hospital OPC with post-concussion syndrome in the form of headache and amnesia which lasted for three months (Fig. 5). 


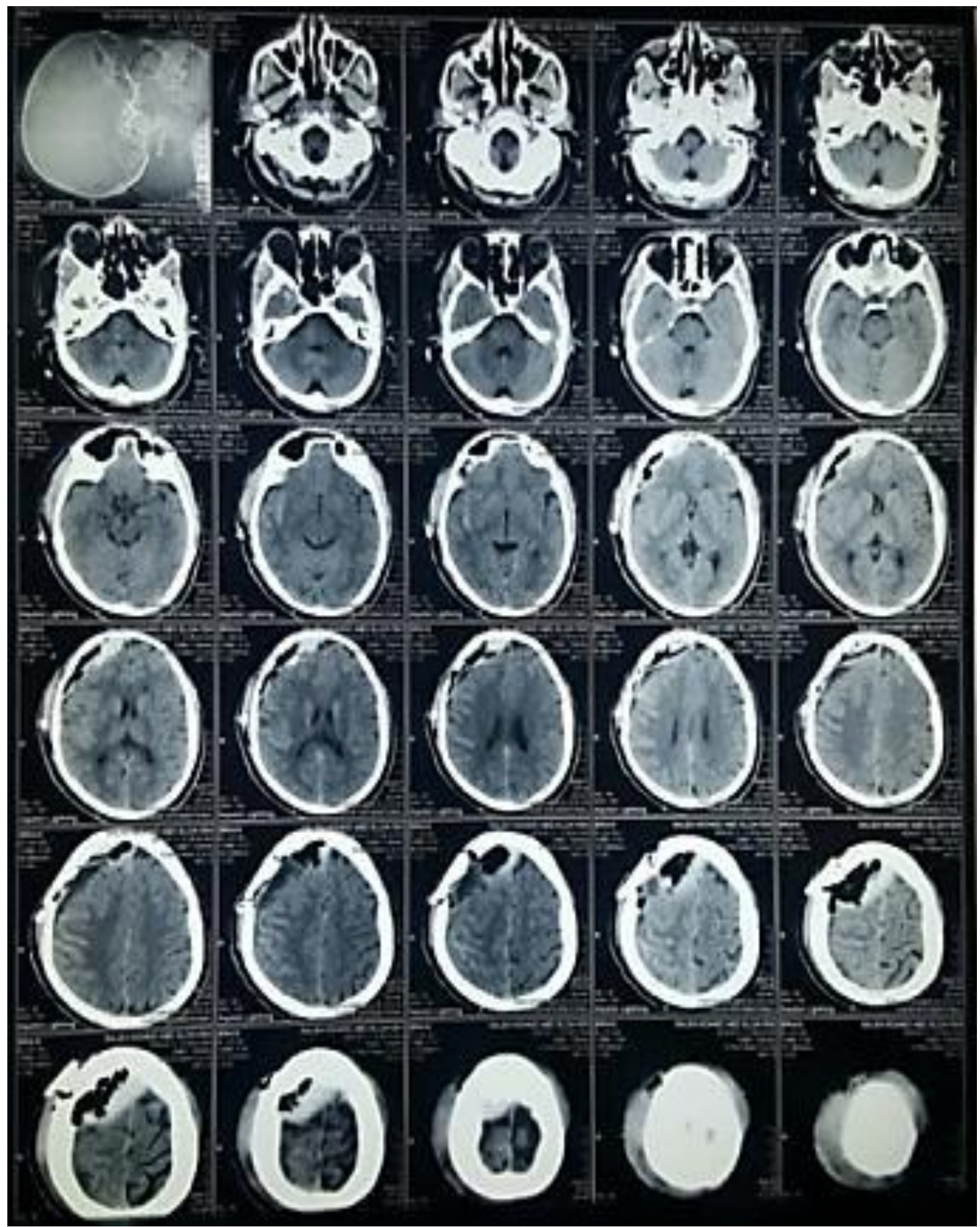

Figure (5): Follow up CT scan 24 hr. post Rt. Frontal massive EDH evacuation. 


\section{CASE 2}

A 35 years old $\lesssim$ patient with history of four hours duration head trauma, No Hx fits nor loss of consciousness. He came to Emergency Room (ER) with GCS 13/15, repeated vomiting, O/E both pupils were regular rounded and reactive and right side weakness 3/5. First aid was done to him, full labs and ABG was normal. CT brain was done which revealed Lt. fronto-tempro-parietal acute SDH (Fig. 6) with $\geq 6 \mathrm{~mm}$ midline shift. Patient was admitted at Neurosurgery Intensive Care Unit. The patient was prepared for surgery. Evacuation of hematoma done for the case by craniotomy.

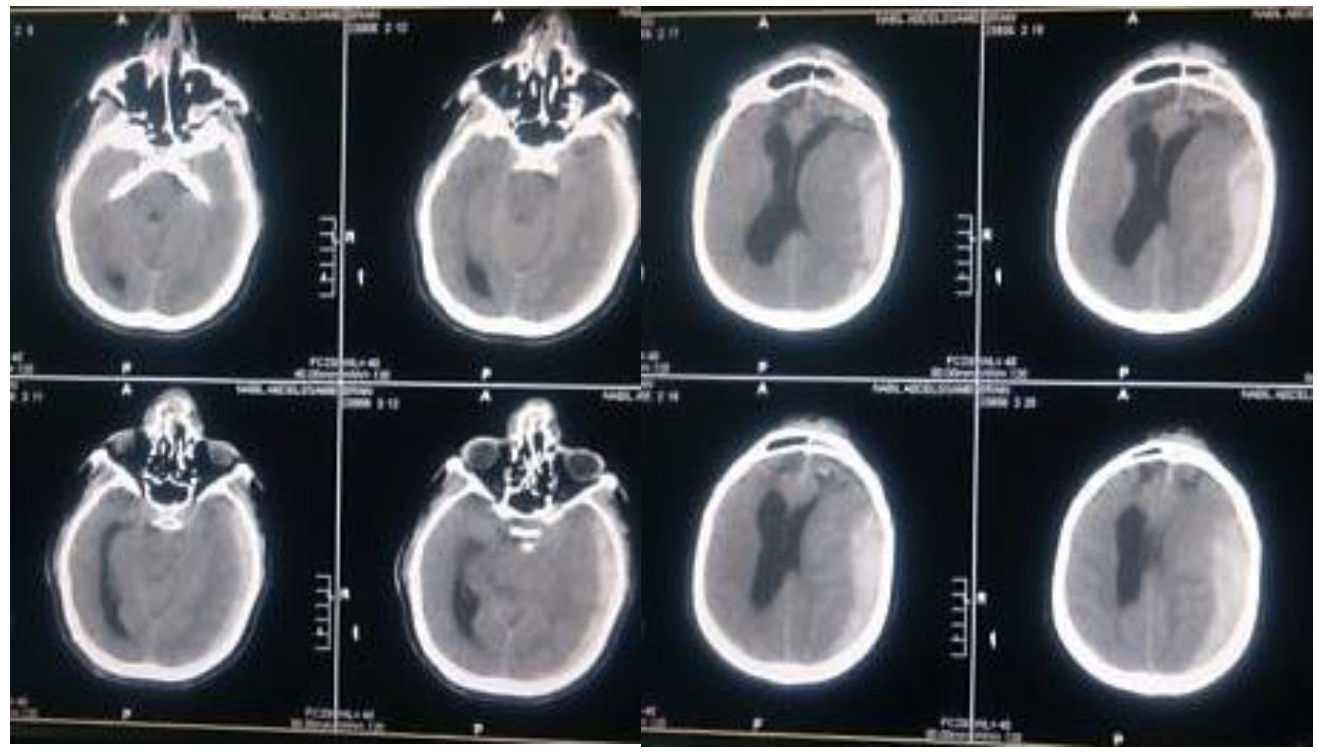

Figure (6): Pre-operative CT Scan Lt. Fronto-tempro-parietal Acute SDH.

After 24 hours follow up CT showed that midline shift was decreased and weakness was improved. Few days later, the patient was discharged fully conscious to complete his medication at home. One month later patient visited our hospital OPC while weakness was still found 2/5. Physiotherapy was recommended with follow up every two weeks. Three month later weakness improved.

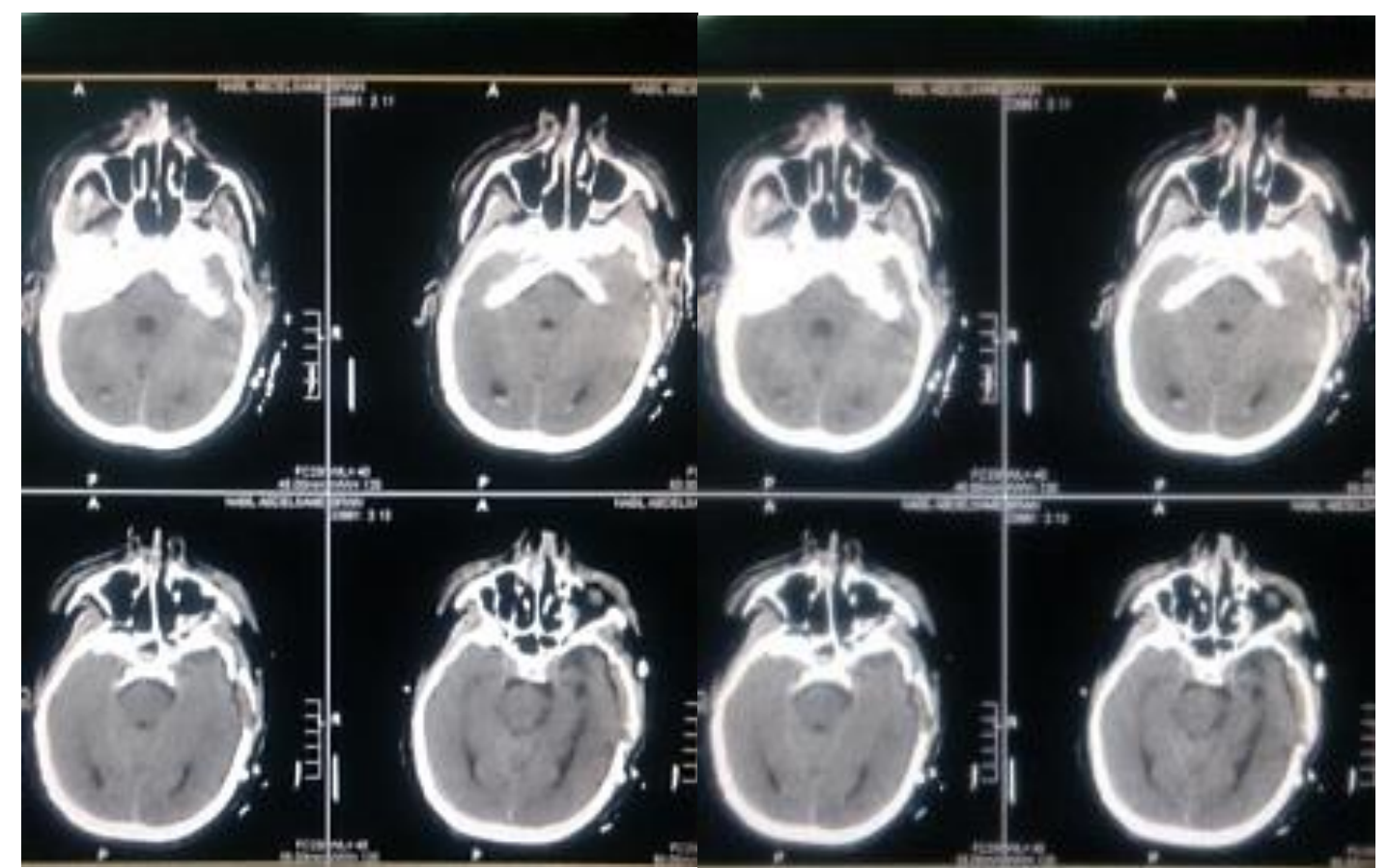

Figure (7): Follow up CT scan 24 hr. post Lt. Fronto-tempro-parietal Acute SDH. 


\section{DISCUSSION}

In the present study, which lasted around 12 month of practical researches on sixty two cases, we found that 22 of our hospital ER cases i.e. 35\% needed surgical interference and the rest was managed conservatively.

According to GCS, the patients were divided into two main groups, pre- and post-operative groups. Each group was subdivided into six sub groups. According to pupil reaction, patients were divided into two groups, pre- and post-operative groups (as above). Each group was divided into two subgroups, reactive pupil and dilated fixed pupil as shown in table (2). The post-operative groups were 21 pts. $(95.5 \%)$ with reactive pupil and only one pt. (4.5\%) with dilated fixed pupil (NB: this case was presented by massive EDH and brain edema leading to brain herniation and death).

Yang et $\boldsymbol{a l} .{ }^{(3)}$ reported that patients were divided into three groups according to their GCS score at admission (GCS 3-5, GCS 6-9, GCS >9). The frequency of complications among the three groups was compared (61.8\% for GCS 3-5, 38.5\% for GCS 6-9, $35.7 \%$ for GCS >9). Our study showed that majority of cases had GCS $\leq 8(95.5 \%)$, while cases of GCS $\geq 8$ $(12.9 \%)$. By comparison between Yang and the present study, the percentage of less complicated cases in present study was $95.5 \%$ while in Yang's study was $12.9 \%$.

According to pupillary reaction in present study, $4.5 \%$ was abnormal and the rest was normal while Murat et al. ${ }^{(4)}$ study reported that $14.2 \%$ of cases were abnormal pupils while the rest were normal. The results suggested that patients with severe head trauma at admission had a significantly higher incidence rate of low GCS as well as complications.

According to post-concussion syndrome, it was positive in 19 cases $(86.36 \%$ ) of the studied 22 cases in the form of headache (18 cases i.e. $81.81 \%$ ), vertigo (15 cases i.e. $68 \%$ ) and loss of consciousness (LOC) at time of trauma (19 cases i.e. 86.36\%). LOC cases were divided as follows: 15 out of 19 pts. (i.e.79 \%) were $\leq$ 10 minutes, 4 out of the 19 studied cases (i.e. $21 \%$ ) were $\geq 10$ minutes. Dizziness were 20 cases $(90.90 \%)$,

According to neurological deficit, 4 cases (18.18\%) showed one case with grade four Rt. side weakness and the rest varied between grades two to three Lt. side weakness. Seizures were 8 cases (36.3\%). The seizures group (7 pts. 87.5\%) improved in the form of decreased antiepileptic drugs doses after about six month while one pt. (12.5\%) didn't improve as the case still on antiepileptic drugs. The pt. with non-improved weakness presented with huge EDH compressing the matter area and came late to our hospital [6hrs after trauma (delayed surgical manner)]. Jourdan et al. ${ }^{(5)}$ reported that headache was $36 \%, 40 \%$ of them lasted for two months then improved. Vertigo was $58.3 \%, 80.5 \%$ of them improved after about three months. Dizziness was $70 \%$, of them $90 \%$ improved after about three months. Neurological deficit was 30\%, $60 \%$ of them totally improved after about six months. Seizures were $14.7 \%, 80 \%$ of them improved after about six months. Blennow et al. ${ }^{(6)}$ reported that $80-90 \%$ of cases represented with post-concussion syndrome. Charlotte (7) study reported that $23 \%$ of PCS cases were fully recovered. In follow up 22\% lasted for 1 month. Cohen (8) study reported that all pts. with headache, dizziness and vertigo suffered from long period of LOC at time of accident, which made them to last longer period of time for recovery than those of the same groups but with shorter time of LOC. Satz ${ }^{(9)}$ study showed that there was no improvement of weakness and seizures.

According to Radiological findings, the majority of lesions were EDH (17 pts. i.e.77.27\%). SDH were 5 cases $(22.72 \%)$ of the studied 22 cases. They were divided as follows: 3 pts. $(60 \%)$ were acute SDH and 2 pts. (40\%) were subacute SDH. EDH were associated with SAH in 4 pts. (23.5\%). 3 of these 4 pts. EDH were also associated with brain edema in 10 pts. (58.8\%) and midline shift in 6 pt. (35.3\%) of the studied 17 cases.

SDH were associated with SAH in 2 pts (40\%), brain edema in 1 pt. $(20 \%)$, and midline shifts in 1 pt. $(20 \%)$ of the studied 5 cases.

SAH presented in Suprasellar Cistern in 4 pt. (18.18\%), all with EDH cases. In the sylvian fissure, 3 pts. (13.63\%) 2 of them with SDH and only 1 with EDH.

Midline shift was found in 7 pts. (31.81\%) of the studied 22 cases. They were distributed as follows according to their amount: $3-4 \mathrm{~mm}$ in 3 pts $(42.85 \%)$, 6$8 \mathrm{~mm}$ in 3 pts $(42.85 \%)$ and $8-11 \mathrm{~mm}$ only in one pt $(14.28 \%)$ of the studied 7 cases.

From the previous data, we found that the GCS of cases associated with $\mathrm{SAH}$, midline shift and brain edema ranged between 13 and 8 .

Murat et al. ${ }^{(4)}$ study reported that the majority of hemorrhage was cases of EDH (45\%), then cerebral contusion cases $(10 \%)$. Waganekar et al. ${ }^{(10)}$ study showed that FU of CT findings was as follows: $80 \%$ of SDH cases, FU CTs were Free, while all FU CTs of EDH cases were free, as well as SAH FU CTs took about four months to improve except for case with midline shift more than $11 \mathrm{~mm}$ which died.

Post-operative FU CT findings were as follows: all SDH CTs were Free, all FU CTs of EDH cases were free except for one case which died three days after operation. SAH FU CTs took about three months to improve except for 3 pts with midline shift more than $6 \mathrm{~mm}$ where they took more than six months to improve.

According to Survival and mortality rates outcome of our studied cases, the survived cases were $21(95.5 \%)$ cases, while one case died $(4.5 \%)$ of the 22 studied cases. From the previous data, one of the causes of death was sever midline shift, which was more than 
$8.5 \mathrm{~mm}$ in this case as well as massive brain edema.

Yang et al. ${ }^{(3)}$ study conducted that the mortality rate was $23.1 \%$ of the studied cases. Jourdan et al. (5) reported that about $10 \%$ of cases TBI was responsible for the worse consequences, with $39 \%$ early mortality rates and $60 \%$ unfavorable outcomes. Charlotte ${ }^{(7)}$ study reported that $97 \%$ of conducted cases survived while the rest died. Jennett ${ }^{(11)}$ study reported that $97 \%$ of conducted cases survived while the rest died.

\section{CONCLUSION}

Early diagnosis and management of lesions of closed head injury is very important to prevent secondary brain injury and to decrease mortality.

\section{REFERENCES}

1. Rangel-Castilla L, Russin JJ, Spetzler RF et al. (2016): Surgical management of skull base tumors. Rep Pract Oncol Radiother., 21 (4): 325-35.

2. Tuong HL, Gean AD (2006): Imaging of Head Trauma. Seminar in Roentgenology, 12: 177-189.

3. Yang XF, Wen L, Shen F, Li G et al. (2008): Surgical complications secondary to decompressive craniectomy in patients with a head injury. https://journals.sagepub.com/doi/abs/10.18679/CN116030_R.2016.008
4. Murat Ö, Ozqur K, Hakan T et al. (2009): Clinical Indicators of Traumatic Brain Injury and Skull Fracture in Pediatric Head Trauma Patients. Turk J Med., 9 (4): 153-158.

5. Jourdan C, Bayen E, Pradat-Diehl P et al. (2016): A comprehensive picture of 4-year outcome of severe brain injuries. Results from the Paris-TBI study. Elsevier Masson Journal, 59: 100-106.

6. Blennow K, David LB, Patrick MK et al. (2016): Traumatic brain injuries. Nat Rev Dis Primers, 2: 1608416088.

7. Charlotte AF (2008): Head injuries in children, incidence, sequel and informational needs. https://pdfs.semanticscholar. org/2e24/ 8529ba 24be1cbe1347d483453fc2fda4d35a.pdf

8. Cohen J (1988): Statistical power analysis for the behavioral science. http://www.utstat.toronto.edu / brunner/oldclass/ 378f16/readings/Cohen Power.pdf

9. Satz P (1993): Brain reserve capacity on symptom onset after brain injury: A formulation and review of evidence for threshold theory. Neuropsychology, 7 (3): 273-295.

10. Waganekar A, Sadasivan J, Prabhu S et al. (2018): Computed Tomography Profile and its Utilization in Head Injury Patients in Emergency Department: A Prospective Observational Study. J Emerg Trauma Shock, 11: 25-30.

11. Jennett B (1996): Epidemiology of head injury. J Neurol Neurosurg Psychiatry, 60 (4): 362-369. 